\section{G95(P) SUDDEN INFANT DEATH SYNDROME AND CAR SEATS: A SYSTEMATIC LITERATURE REVIEW}

${ }^{1}$ I Plumptre, ${ }^{2} \mathrm{C}$ Phillips, ${ }^{3} \mathrm{~S}$ Williams, ${ }^{4} \mathrm{C}$ Plumptre. ${ }^{1}$ Department of Paediatrics, London North West Healthcare NHS Trust, London, UK; ${ }^{2}$ Acute Medical Unit, King's College NHS Trust, London, UK; ${ }^{3}$ Department of Rheumatology, Guy's and St Thomas' Trust, London, UK; ${ }^{4}$ Medical Sciences Division, University of Oxford, Oxford, UK

\subsection{6/archdischild-2018-rcpch.92}

Aims Sudden infant death syndrome (SIDS) was responsible for 191 infant deaths in England and Wales in 2015. ${ }^{1}$ SIDS rates have declined substantially in recent years, thanks to successful campaigns about known modifiable risk factors such as smoking in pregnancy and sleeping positions. ${ }^{2}$ Guidance about at-home sleeping to reduce SIDS (such as avoidance of co-sleeping, bedding and temperature) is widely available. However, guidance about safe out-of-home sleeping, for example in buggies or car seats, is more limited. We aimed to assess the evidence about the role of car seats in SIDS.

Methods We conducted a systematic literature review in PubMed using the search terms 'sudden infant death syndrome AND car seat' or 'SIDS AND car seat'. Article abstracts were screened for relevance. Articles discussing infant death in a car seat from an explained cause were excluded.

Results A systematic literature review revealed 10 results. Of these, abstract screening excluded 7 results for describing booster seats $(n=2)$, and explained deaths such as injury or hanging $(n=5)$. Of included articles, one retrospective review of 11717 infant deaths identified that out-of-home SIDS were 2.6 times more likely to be associated with a carseat or stroller. ${ }^{3}$ A second retrospective review highlighted 14 car seat associated unexplained infant deaths out of 1465 autopsies, 10 of which were outside of travel use. ${ }^{4}$ This supports recommendations that car seats should be used for travel only. ${ }^{5} 3$ further articles identified from references highlighted work demonstrating reduced oxygen saturations in infants in simulated moving car seats. ${ }^{6}$ This supports a separate finding that attributed $48 \%$ of car seat associated deaths to positional asphyxia. ${ }^{7}$ Longer periods in car seats also increase the risk of airway obstruction. ${ }^{8}$

Conclusions Although car seats are an important legal requirement, ${ }^{9}$ evidence suggests that prolonged periods in a car seat are dangerous for infants. However, there is a very limited evidence base for guidelines on exactly how long car seats should be used in infants. There is an urgent need for further research into the interplay of car seats and SIDS in order to produce practical advice for parents.

\section{REFERENCES}

1. Office of National Statistics. Unexplained deaths in infancy: England and Wales, 20102015 August; $1-13$.

2. The Lullaby Trust. Safer sleep advice. https://www.lullabytrust.org.uk/safer-sleepadvice/

3. Kassa H, Moon RY, Colvin JD. Risk factors for sleep-related infant deaths in inhome and out-of-home settings. Paediatrics 2016:138(5):e20161124e20161124. doi:10.1542/peds.2016-1124

4. Bamber AR, Pryce J, Ashworth MT, Sebire NJ. Sudden unexpected infant deaths associated with car seats. Forensic Sci Med Pathol 2014;10(2):187-192. doi:10.1007/s12024-013-9524-5

5. Committee on Injury and Poison Prevention and Committee on Fetus and Newborn. Safe Transportation of Premature and Low Birth Weight Infants. Pediatr 1996;97(5):758-760.
6. Arya R, Williams G, Kilonback $A$, et al. Is the infant car seat challenge useful? A pilot study in a simulated moving vehicle. Arch Dis Child: Fetal Neonatal Ed 2017;102(2):F136-F141. doi:10.1136/archdischild-2016-310730

7. Batra EK, Midgett JD, Moon RY. Hazards associated with sitting and carrying devices for children two years and younger. I Pediatr 2015;167(1):183-187. doi:10.1016/i.jpeds.2015.03.044

8. Cote A, Bairam A, Deschenes M, Hatzakis G. Sudden infant deaths in sitting devices. Arch Dis Child 2008;93(5):384-389. doi:10.1136/adc.2007.119180

9. Child car seats: the law. https://www.gov.uk/child-car-seats-the-rules

\section{G96(P) ARE WHITE CELLS IN CHILDREN'S URINE DIAGNOSTIC OR A DISTRACTION, AND DOES COLLECTION AND CULTURE METHOD MATTER? NEW DATA FROM 4910 ACUTELY UNWELL CHILDREN}

${ }^{1} \mathrm{~S}$ Alam, ${ }^{1} \mathrm{~J}$ van der Voort, ${ }^{2} \mathrm{M}$ Wootton, ${ }^{1} \mathrm{~B}$ Creswell, ${ }^{3} \mathrm{~A}$ Hay, ${ }^{4} \mathrm{C}$ Butler. ${ }^{1}$ Children's Hospital for Wales, University Hospital of Wales, Cardiff, UK; ${ }^{2}$ Specialist Antimicrobial Chemotherapy Unit, University Hospital of Wales, Cardiff, UK; ${ }^{3}$ Centre for Academic Primany Care, NIHR School for Primary Care Research, University of Bristol, Bristol, UK; ${ }^{4}$ Nuffield Department of Primary Care Health Sciences, University of Oxford, Oxford, UK

\subsection{6/archdischild-2018-rcpch.93}

Aims To describe the relationship between urine white cell count (WCC) and the microbiological diagnosis of UTI in acutely unwell children, and explore the influence of culture and collection method.

Methods Multi-centre, prospective, cohort study of acutely unwell children aged 3 months-5 years presenting to UK primary care whose urine samples were collected by clean catch or nappy pad, and cultured by both a Central Laboratory (CL) using spiral-plating, and Local NHS Laboratories (LL) using standard practice. Positive and negative predictive values (PPV and NPV) of diagnosing UTI were calculated for WCC $\geq 100 / \mathrm{mm}^{3}$ and WCC $\leq 10 / \mathrm{mm}^{3}$ respectively, comparing laboratories and sampling methods.

Results Of 4910 samples, 1.9\% had UTI in the CL and 5.3\% in the LL.

Table 1 outlines the PPV of WCC $\geq 100 / \mathrm{mm}^{3}$ and NPV of WCC $\leq 10 / \mathrm{mm}^{3}$ for diagnosing UTI by laboratory culture and sampling method.

\begin{tabular}{lllllll}
\multicolumn{7}{l}{ Abstract G96(P) Table 1 } \\
\hline & \multicolumn{7}{l}{ CL culture } \\
\cline { 2 - 7 } & All & Clean & Nappy & All & Clean & Nappy \\
& Samples & Catch & Pad & Samples & Catch & Pad \\
\hline PPV of & $23.9 \%$ & $33.3 \%$ & 0 & $30.3 \%$ & $38.7 \%$ & $6.9 \%$ \\
WCC $\geq 100 / \mathrm{mm}^{3}$ & {$[17.5-$} & {$[25.0-$} & & {$[22.7-$} & {$[29.3-$} & {$[1.8-$} \\
& $31.6 \%]$ & $42.8 \%]$ & & $39.1 \%]$ & $49.0 \%]$ & $23.6 \%]$ \\
NPV of & $99.0 \%$ & $99.3 \%$ & $98.6 \%$ & $95.8 \%$ & $97.7 \%$ & $93.6 \%$ \\
WCC $\leq 10 / \mathrm{mm}^{3}$ & {$[98.7-$} & {$[98.9-$} & {$[98.3-$} & {$[95.4-$} & {$[97.2-$} & {$[92.9-$} \\
& $99.2 \%]$ & $99.5 \%]$ & $98.9 \%]$ & $96.2 \%]$ & $98.2 \%]$ & $94.2 \%]$ \\
\hline
\end{tabular}

Conclusions Local NHS Labs diagnose almost $3 \mathrm{x}$ more UTIs than a reference Central Laboratory. Many of these may be false positives.

A WCC $\geq 100 / \mathrm{mm}^{3}$ poorly predicts UTI on culture in acutely unwell young children consulting in primary care.

Despite WCC $\leq 10 / \mathrm{mm}^{3}$ having a good NPV regardless of collection or culture method, 37 of the 92 positive CL cultures (40\%) had WCC $\leq 10 / \mathrm{mm}^{3}$.

Recommendation We propose a diagnostic algorithm selectively utilising spiral-plating culture methods to potentially minimise 
unnecessary treatment and investigation for 9000 children per year across England and Wales.

\section{G97(P) ADOLESCENT OBESITY: EXPLORING THE HEALTH RELATED QUALITY OF LIFE IN HELP SEEKING OBESE ADOLESCENTS IN THE UNITED KINGDOM}

AR Rolle. Institute of Child Health, University College of London, London, UK

\subsection{6/archdischild-2018-rcpch.94}

Background According to previous studies, overweight and obesity in children and adolescents were associated with impaired health-related quality of life (HRQOL) and depression. The objective of this study is to describe the HRQOL and risk for depression in help seeking obese adolescents within the United Kingdom.

Methods This is a cross-sectional study conducted among 293 obese adolescents (13-18 years) attending the weight management clinic at the University College of London Hospital, England. The stratified cluster sampling framework was used; all participants were referred to this clinic by a general practitioner or paediatrician. Body Mass Index (BMI) was calculated using BMI z-score. The Paediatric Quality of Life Questionnaire (PedsQL) was used to measure HRQOL and The Short Mood and Feeling Questionnaire (SMFQ) was used to assess the likelihood of depression.

Results Study included 123 males and 170 females, mean BMI 43. Findings revealed that a higher quality of life (QOL) was found among younger adolescents, with lower QOL found among obese individuals. Female adolescents were found to score lower on emotional functioning and depression when compared with males, and adolescents who met the threshold for depression scored lower on all measures relating to QOL. The study population had a lower QOL of life when compared to healthy adolescents, and paediatric population with chronic diseases such as asthma, diabetes, and cancer.

Conclusion Compared with healthy adolescent population, the QOL in the study population was lower, and adolescents who met the threshold for depression had an overall lower QOL.

\section{Trainees session}

\section{G98 EXCEPTION REPORTING AT GREAT ORMOND STREET HOSPITAL - BUILDING ON JUNIOR DOCTORS EXPERIENCES}

${ }^{1} G L P$ Manning, 'D Das, 1J Hassell, 1,2E Parish, 'S Ottaway, 1,2J Poisson, 1,2S Sharma, ${ }^{1} \mathrm{R}$ McCulloch. ${ }^{1} J u n i o r$ Doctors Forum, Great Ormond Street Hospital, London, UK; ${ }^{2}$ Post Graduate Medical Education, Great Ormond Street Hospital, London, UK

\subsection{6/archdischild-2018-rcpch.95}

Aims The new English/Welsh Junior Doctor (JD) contract introduces a powerful tool for positive change - the Exception Report (ER). Following a staged introduction, ER has been available to all JDs (including those not in training), throughout Great Ormond Street Hospital (GOSH) since June 2017. Initial rates of ER have been low, with concerns of JD disillusionment. This project aims to improve the number of submitted ERs with secondary analysis of ER outcome.

Methods A process map identified primary drivers of reporting; Educational Supervisor (ES) engagement; and systemic change. Access to ER systems was improved by incorporating with the postgraduate medical education (PGME) smartphone app. ER was decoupled from ES by allocating departmental ER consultant leads. Ideas on local exception causes; barriers to reporting; and potential solutions were obtained from a JD focus group, which aided production of a guideline for ER leads to address ER root causes. After promoting ER at hospital induction, further interventions were targeted by surveying all JDs on personal experience of exceptions; ER processes; and opinions on reporting.

Progress was measured by graphing ER numbers, broken down by reporting department. Future change ideas included a PGME-led awareness campaign, including website, e-newsletter, $(\mathrm{P})$, video and podcast facets; publicity of previous work on JD perspectives; creation of a framework, targeted at JDs, encouraging junior-lead solutions when reporting; supplementation of PGME ES resources; and targeted signposting towards currently-available courses and learning resources.

Results 56 ERs were submitted during Mar-Sep 2017, leading to plans to redesign one department's rota, with financial compensation for three JDs in multiple departments. An additional fellow is being recruited into another department following a JD-initiated business case including, amongst other arguments, ER submissions. No fines have been levied. Multiple interventions are ongoing, with monthly plan-do-study-act (PDSA) cycles planned to aid continued improvement, and to ensure all JDs at GOSH feel empowered to submit ERs.

Conclusions ER should be used positively to identify system issues, and early usage at GOSH has demonstrated ER to both support and initiate constructive departmental change. Impact from current interventions is awaited, as departments harness the potential of the ER process.

\section{G99 SUPPORTING PAEDIATRIC TRAINEES INVOLVED IN MEDICO-LEGAL INVESTIGATIONS}

A Sammut, W Kelsall. Neonatal Intensive Care Unit, Cambridge University Hospitals NHS Foundation Trust, Cambridge, UK

\subsection{6/archdischild-2018-rcpch.96}

Aim Feedback from trainees highlighted different levels of support offered to trainees across the training programme. Many trainees have limited experience of Coroner's inquests, Court cases, GMC fitness to practice hearings, CQC visits, statement writing, serious incident investigations, Deanery and School of Paediatrics visits. More junior trainees have described these as stressful events. The aim of this study was to review the support offered to trainees and develop more consistent approaches.

Methods An online regional trainee survey was conducted between October 2016 and January 2017. This included 8 multiple choice questions with free text comments. Questions related to whether (1) trainees had been involved in such events during their training, (2) which trusts they worked in, (3) whether they received adequate support, and (4) what training if any, they had in these areas. The web link was sent to all trainees by the Paediatric Specialty Administrator and posted on the School Facebook Group.

Results A total of 40 paediatric trainees replied. 34 (85\%) reported previous involvement in such events: 20 (59\%) felt well supported by their hospital, 8 (24\%) felt that they needed support but that this was not offered or available locally and $6(17 \%)$ felt 